\title{
Statistical structure of the biological tissue scattering of laser field with the complex degree of coherence
}

\author{
P.O. Angelsky \\ Yuriy Fedkovych National University of Chernivtsi, \\ Department of Editing and Printing Art, \\ 2, Kotsiubynskyi str., 58001 Chernivtsi, Ukraine
}

\begin{abstract}
Diagnostic efficiency of differentiation of birefringence of rat's biological tissues in different physiological states is studied in this research based on application of the correlation approach. Interrelation between coordinate distributions of complex degree of coherence (CDC) and changes in birefringence has been determined.
\end{abstract}

Keywords: biological tissue, complex degree of coherence, laser light scattering.

Manuscript received 22.05.14; revised version received 05.08.14; accepted for publication 29.10.14; published online 10.11.14.

\section{Introduction}

New directions in investigation of scattered radiation fields have been recently formed - correlation optics [1] and singular optics [2] - which became the foundation for formation of laser polarimetry of $2 \mathrm{D}$ digital images of optically anisotropic phase inhomogeneous layers of biological origin [3].

Two main groups of laser polarimetry techniques are distinguished, namely: "one-point" (determination of polarization parameters distribution in the points of the image) and "two-point" (determination of the degree of coherence between polarization states of spatially distant points) ones [4-9].

Besides, under the condition of multiple scattering, the polarization information about optically anisotropic structure of the object is averaged and loses its unilateral diagnostic sense. However, the coordinate phase distributions of the fields of scattered radiation remain informative.

Thus, development and substantiation of principles of the correlation approach to description of laser radiation fields multiply scattered by the layers of biological tissues on the basis of statistical analyzing the coordinate distributions of values inherent to the modulus of complex degree of coherence is topical.

\section{Experimental technique}

In this research, the distributions of CDC for the field of laser radiation transformed by optically thick layers (attenuation coefficient $\tau=0.75$ ) of histological sections of skeleton muscle tissue of healthy rats and that of rats with septic inflammation were investigated.

Fig. 1 presents the optical setup for phasometry of laser images describing nets of biological crystals.

Illumination was performed by the parallel beam $(\varnothing=$ $\left.10^{4} \mu \mathrm{m}\right)$ of He-Ne laser $(\lambda=0.6328 \mu \mathrm{m}) 1$. By means of polarization light source (quarter-wave plates 3, 5 and polarizer 4 ) the right-hand circularly polarized beam was formed. The images of the layers of biological tissues or fluids 6 were projected by the micro-objective 7 in the plane of light-sensitive plate $(800 \times 600)$ of CCD camera 10 .

The technique for experimental measurements of the above mentioned correlation-phase parameter was as follows: 


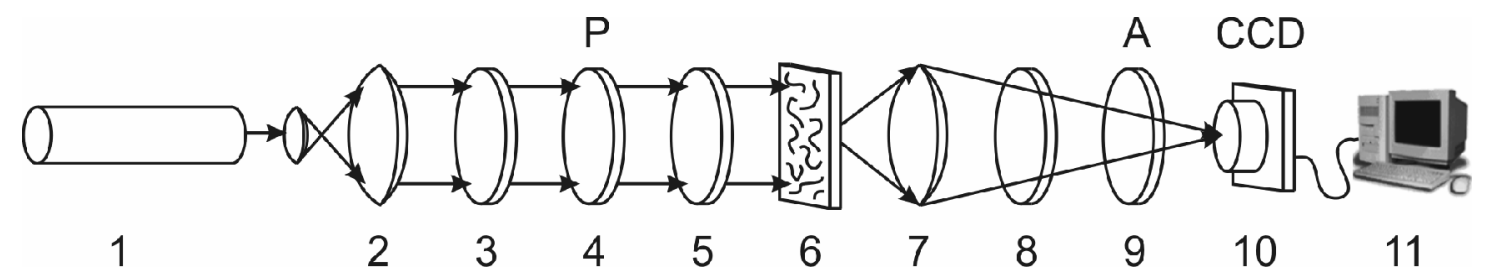

Fig. 1. Optical setup of phasometer: 1 - He-Ne laser; 2 - collimator; 3, 5, 8- quarter-wave plates; 4, 9-polarizer and analyzer, respectively; 6 - object of investigation; 7 - micro-objective; 10 - CCD camera; 11 - personal computer.

1. The transmission plane of the analyzer 8 was successively oriented at the angles $\Theta=0^{\circ}, \Theta=90^{\circ}$, and the corresponding sets of intensity values $I^{(0)}\left(\begin{array}{l}r_{11}, \ldots r_{1 m} \\ \ldots \ldots \ldots . \\ r_{n 1}, \ldots . . \\ r_{n m}\end{array}\right), I^{(90)}\left(\begin{array}{l}r_{11}, \ldots . . r_{1 m} \\ \ldots \ldots \ldots . \\ r_{n 1}, \ldots . . r_{n m}\end{array}\right)$ were measured.

2. The sets of values of orthogonal components of the amplitude of laser radiation

$$
\begin{aligned}
& E_{x}\left(\begin{array}{l}
r_{11}, \ldots r_{1 m} \\
\ldots \ldots . . . \\
r_{n 1}, \ldots r_{n m}
\end{array}\right)=\sqrt{I^{(0)}\left(\begin{array}{l}
r_{11}, \ldots r_{1 m} \\
\ldots \ldots \ldots . \\
r_{n 1}, \ldots . r_{n m}
\end{array}\right)} \text { and }
\end{aligned}
$$

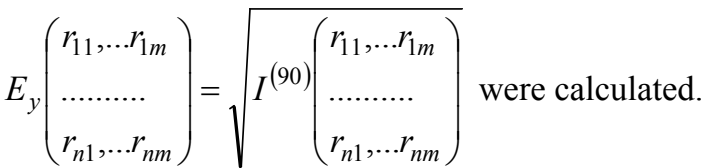

3. By rotating the transmission planes of polarizer and analyzer using the classical technique suggested in $[139,148]$, the set of values of the Jones matrix elements $d_{i k}\left(\begin{array}{l}r_{11}, \ldots r_{1 m} \\ \ldots \ldots . . . \\ r_{n 1}, \ldots r_{n m}\end{array}\right)$ was determined.

4. The correlation-phase map of the laser field scattered by the layer of biological tissue was calculated using the following relations:

$$
\begin{aligned}
& \mu(\Delta r)=\frac{Q_{12}\left(r_{1}, r_{2}\right) M_{12}\left(r_{1}, r_{2}\right)}{Q_{11}\left(r_{1}, r_{1}\right) Q_{22}\left(r_{2}, r_{2}\right)}, \\
& Q_{12}\left(r_{1}, r_{2}\right)=\left(\sqrt{I^{(0)}\left(\begin{array}{l}
r_{11}, \ldots r_{1 m} \\
\ldots \ldots \ldots . \\
r_{n 1}, \ldots r_{n m}
\end{array}\right)} \times \sqrt{I^{(0)}\left(\begin{array}{l}
r_{11}+\Delta r, \ldots r_{1 m}+\Delta r \\
\ldots \ldots \ldots . . . \\
r_{n 1}+\Delta r, \ldots r_{n m}+\Delta r
\end{array}\right)}\right)+
\end{aligned}
$$

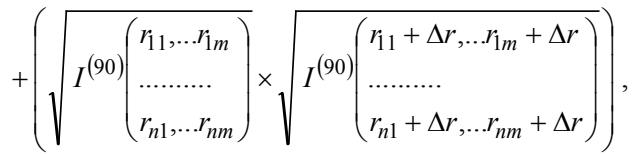

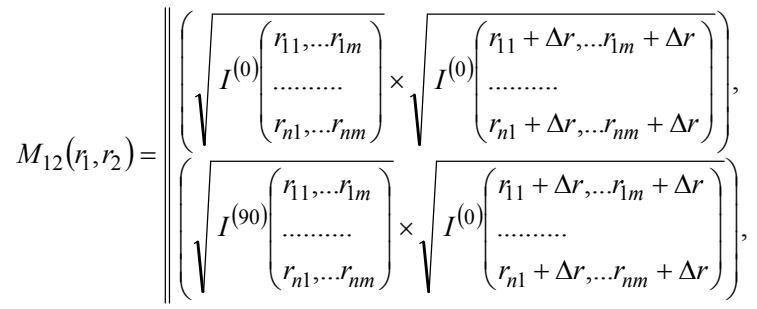

\section{(C) 2014, V. Lashkaryov Institute of Semiconductor Physics, National Academy of Sciences of Ukraine}

$$
\begin{aligned}
& Q_{11}\left(r_{1}, r_{1}\right)=I^{(0)}\left(\begin{array}{l}
r_{11}, \ldots r_{1 m} \\
\ldots \ldots \ldots . . . \\
r_{n 1}, \ldots r_{n m}
\end{array}\right)+I^{(90)}\left(\begin{array}{l}
r_{11}, \ldots r_{1 m} \\
\ldots \ldots \ldots . \\
r_{n 1}, \ldots r_{n m}
\end{array}\right), \\
& Q_{22}\left(r_{2}, r_{2}\right)=I^{(0)}\left(\begin{array}{l}
r_{11}+\Delta r, \ldots r_{1 m}+\Delta r \\
\ldots \ldots \ldots \\
r_{n 1}+\Delta r, \ldots . .
\end{array}\right)+ \\
& +I_{n m}^{(90)}\left(\begin{array}{l}
r_{11}+\Delta r, \ldots r_{1 m}+\Delta r \\
\ldots \ldots \ldots \\
r_{n 1}+\Delta r, \ldots r_{n m}+\Delta r
\end{array}\right)
\end{aligned}
$$

Here, $\Delta r$ is the step of coordinate scanning by rows

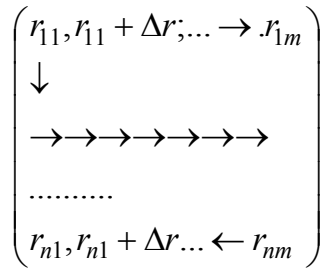

of $2 \mathrm{D}$ array of values inherent to

the complex degree of mutual coherence $\mu\left(\begin{array}{l}r_{11}, \ldots r_{1 m} \\ \ldots \ldots \ldots . . . \\ r_{n 1}, \ldots . r_{n m}\end{array}\right)$ for the field of scattered laser radiation.

\section{Experimental results and discussion}

Fig. 2 shows the distributions and histograms of CDC for laser radiation field multiply scattered by the layers of healthy (upper fragment) and inflamed (lower fragment) tissue of the rat's skeletal muscle.

The experimentally determined histograms $H\left(\mu_{12}\right)$ of CDC distributions are asymmetric dependences with several prevailing extremes $\left(\mu_{12}=0.7, \quad \mu_{12}=0.9\right.$, $\mu_{12}=0.82$ ). The values of such local extremes are by 2 ...7 times higher than the probability of other values of $\mu_{12}$ over the whole range of its change $\mu_{12}=0 \ldots 1$. 


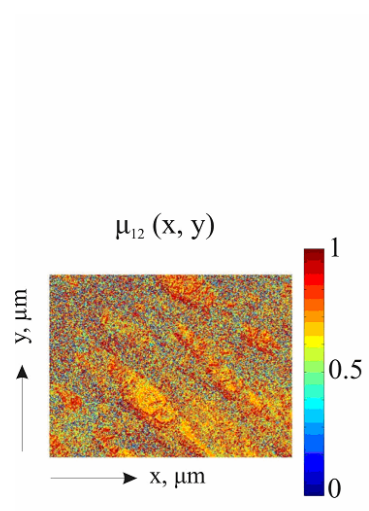

(a)

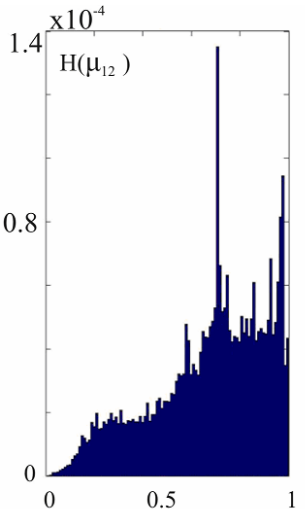

(b)

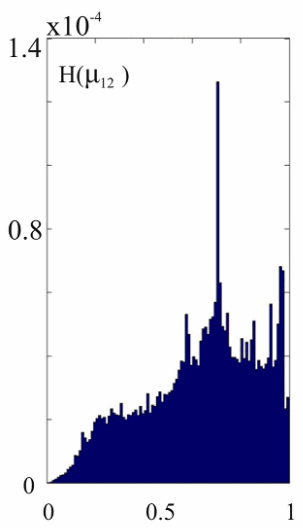

(d) (c)

Fig. 2. Coordinate (left column) and probability (right column) distributions of the degree of mutual coherence for laser radiation transformed by optically thick layers of healthy (upper fragment) and inflamed (lower fragment) tissue of the rat's skeletal muscle.

The comparative analysis of histograms $H\left(\mu_{12}\right)$ characterizing the CDC distributions of object fields of the healthy and inflamed tissues showed the decrease (by $15 \%)$ of the value of the main extremum $\left(\mu_{12}=0.7\right)$ and, on the contrary, the increase (by $35 \%$ and $20 \%$ ) of local $\left(\mu_{12}=0.9, \mu_{12}=0.82\right)$ extremes of this parameter. In our opinion, this transformation is related with the growth of birefringence level for the substance of the muscle tissue with septic inflammation and corresponding formation of structures with strong phase fluctuations.

Table presents quantitative parameters characterizing the coordinate distributions of CDC of laser radiation fields.

The obtained results of investigation aimed at the statistical structure of coordinate distributions of $\mu_{12}(\Delta r)$ revealed the changes in optical anisotropy observed in the sepsis-inflamed tissue of the skeletal muscle, which are manifested in the decrease of the asymmetry value $R_{3}^{\mu}$ (by 9.1 times) and excess $R_{4}^{\mu}$ (by 7.5 times) characterizing the coordinate distributions $\mu_{12}(\Delta r)$.
Table. Statistical moments $R_{i=1-4}^{\mu}$ characterizing distributions $\mu(x, y)$ of the field of muscle tissue in different physiological states.

\begin{tabular}{|c|c|c|}
\hline$R_{i=1-4}^{\mu}$ & Norm & Sepsis \\
\hline$R_{1}^{\mu}$ & $0.67 \pm 0.078$ & $0.71 \pm 0.082$ \\
\hline$R_{2}^{\mu}$ & $0.23 \pm 0.031$ & $0.21 \pm 0.033$ \\
\hline$R_{3}^{\mu}$ & $2.04 \pm 0.27$ & $0.23 \pm 0.031$ \\
\hline$R_{4}^{\mu}$ & $9.36 \pm 1.02$ & $1.21 \pm 0.17$ \\
\hline
\end{tabular}

\section{Conclusions}

1. Diagnostic efficiency of the analysis of phase anisotropy inherent to biological tissues with different optical thickness by applying the statistical analysis of coordinate distributions for the complex degree of coherence of laser radiation scattered by these tissues has been experimentally substantiated.

2. It has been determined that the physical reason of the decrease in asymmetry and excess characterizing the coordinate distributions of the values of coherence complex degree for laser radiation field multiply scattered by optically thick layers of biological tissues consists in the increase of birefringence of the networks of protein crystals. On this basis, the early (pre-clinical) diagnostics of the septic inflammatory process of the rat's muscle tissue has been performed for the first time.

\section{References}

1. V. Sankaran, M.J. Everett, D.J. Maitland, J.T. Walsh, Comparison of polarized-light propagation in biological tissue and phantoms // Opt. Lett. 24, p. 1044-1046 (1999).

2. M.R. Ostermeyer, D.V. Stephens, L. Wang and S.L. Jacques, Nearfield polarization effects on light propagation in random media // Trends in Optics and Photonics: Biomedical Optical Spectroscopy and Diagnostics, 3, p. 20-26 (1996).

3. E. Wolf, Coherence properties of partially polarized electromagnetic radiation // Nuovo Cimento, 13, p. 1165-1181 (1959).

4. P.O. Angelsky, Fourier phasometry of human effusion polycrystalline networks // Appl. Opt. 51(10), p. C70-C76 (2012).

5. P.O. Angelsky, Fourier phasometry of polarization filtered images of biological polycrystalline networks // Optical Memory and Neural Networks, 21(3), p. 192-203 (2012). 
6. P.O. Angelsky, A.G. Ushenko, A.V. Dubolazov, M.I. Sidor, G.B. Bodnar, G. Koval and L. Trifonyuk, The singular approach for processing polarization-inhomogeneous laser images of blood plasma layers // J. Opt. 15, 044030 (8 pages) (2013).

7. A.G. Ushenko, P.O. Angelsky, A.V. Dubolazov et al., Complex polarization-phase and spatialfrequency selections of laser images for blood plasma films in diagnostics of changes in their polycrystalline structure // Optika i spektroskopia, 115(4), p. 681-690 (2013), in Russian.
8. P.O. Angelsky, L.B. Bodnar, M.P. Gorskii, L.Ya. Kushnerick, Fourier-Stokes polarimetry of fields scattered by birefringent biological networks // Optical Memory and Neural Networks, 21(4), p. 233-241 (2012).

9. A.G. Ushenko, P.O. Angelsky, M. Sidor, Yu.F. Marchuk, D.R. Andreychuk, and N.V. Pashkovskaya, Spatial-frequency selection of complex degree of coherence of laser images of blood plasma in diagnostics and differentiation of pathological states of human organism of various nosology // Appl. Opt. 53(10), p. B172-B180 (2014). 